\title{
Front Matter: Volume 9286
}

, "Front Matter: Volume 9286," Proc. SPIE 9286, Second International Conference on Applications of Optics and Photonics, 928601 (22 August 2014); doi: 10.1117/12.2075361

SPIE Event: Second International Conference on Applications of Optics and Photonics, 2014, Aveiro, Portugal 


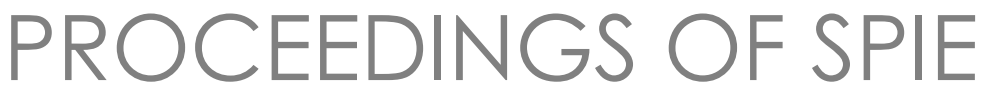

\title{
Second International Conference on Applications of Optics and Photonics
}

\author{
Manuel Filipe P. C. Martins Costa \\ Rogério Nunes Nogueira \\ Editors
}

26-30 May 2014

Aveiro, Portugal

Organized by

SPOF-Portuguese Society for Research and Development of Optics and Photonics (Portugal)

Supported by

IT-Instituto de Telecomunicações (Portugal) • OSA Student Chapter of University of Aveiro ICO-International Commission for Optics • SPIE • EOS-European Optical Society The Optical Society • RIAO—Red Iberoamericana de Óptica • Photonics21_European Technology Platform for Photonics $\bullet$ SEDOPTICA—Sociedad Española de Óptica AMO-Academia Mexicana de Óptica • RCO_Red Colombiana de Óptica STO-Société Tunisienne d'Optique • OPSS-Optics and Photonics Society of Singapore CVO_Comité Venezolano de Optica • Fábrica-Centro Ciência Viva de Aveiro University of Porto SPIE Student Chapter $\bullet$ University of Aveiro • University of Minho EFTA—Esc. F.P. Turismo de Aveiro • City of Aveiro • Innova Scientific • Quantel Laser Micron Optics, Inc. • M.T. Brandão Spectra-Physics • FiberSensing, Sistemas Avançados de Monitorização, S.A. • MWTechnologies, Ltd. • Espectral Telecomunicações, S. A. Tektronix, Inc • Keithley Instruments, Inc.

Volume 9286 
The papers included in this volume were part of the technical conference cited on the cover and title page. Papers were selected and subject to review by the editors and conference program committee. Some conference presentations may not be available for publication. The papers published in these proceedings reflect the work and thoughts of the authors and are published herein as submitted. The publisher is not responsible for the validity of the information or for any outcomes resulting from reliance thereon.

Please use the following format to cite material from this book:

Author(s), "Title of Paper," in Second International Conference on Applications of Optics and Photonics, edited by Manuel Filipe P. C. Martins Costa, Rogério Nunes Nogueira, Proceedings of SPIE Vol. 9286 (SPIE, Bellingham, WA, 2014) Article CID Number.

ISSN: 0277-786X

ISBN: 9781628413618

Published by

SPIE

P.O. Box 10, Bellingham, Washington 98227-0010 USA

Telephone +1 3606763290 (Pacific Time) · Fax +1 3606471445

SPIE.org

Copyright @ 2014, Society of Photo-Optical Instrumentation Engineers.

Copying of material in this book for internal or personal use, or for the internal or personal use of specific clients, beyond the fair use provisions granted by the U.S. Copyright Law is authorized by SPIE subject to payment of copying fees. The Transactional Reporting Service base fee for this volume is $\$ 18.00$ per article (or portion thereof), which should be paid directly to the Copyright Clearance Center (CCC), 222 Rosewood Drive, Danvers, MA 01923. Payment may also be made electronically through CCC Online at copyright.com. Other copying for republication, resale, advertising or promotion, or any form of systematic or multiple reproduction of any material in this book is prohibited except with permission in writing from the publisher. The CCC fee code is 0277-786X/14/\$18.00.

Printed in the United States of America.

Publication of record for individual papers is online in the SPIE Digital Library.

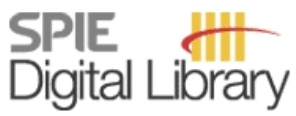

SPIEDigitalLibrary.org

Paper Numbering: Proceedings of SPIE follow an e-First publication model, with papers published first online and then in print and on CD-ROM. Papers are published as they are submitted and meet publication criteria. A unique, consistent, permanent citation identifier (CID) number is assigned to each article at the time of the first publication. Utilization of CIDs allows articles to be fully citable as soon as they are published online, and connects the same identifier to all online, print, and electronic versions of the publication. SPIE uses a six-digit CID article numbering system in which:

- The first four digits correspond to the SPIE volume number.

- The last two digits indicate publication order within the volume using a Base 36 numbering

system employing both numerals and letters. These two-number sets start with 00, 01, 02, 03, 04, $05,06,07,08,09,0 A, 0 B \ldots$. 0Z, followed by 10-1Z, 20-2Z, etc.

The CID Number appears on each page of the manuscript. The complete citation is used on the first page, and an abbreviated version on subsequent pages. Numbers in the index correspond to the last two digits of the six-digit CID Number. 


\title{
Contents
}

\author{
xi Authors \\ $\mathrm{xV} \quad$ Conference Committees \\ xxi Introduction
}

MO.1.A

928605 Photonic integrated transmitter and receiver for NG-PON2 [9286-221]

928606 Neuromorphic opto-electronic integrated circuits for optical signal processing [9286-41]

928607 Calculation of the number of bits required for the estimation of the bit error ratio [9286-128]

928608 Analysis and simulation of ring resonators for slow and fast light devices applications [9286-138]

MO.1.B

928609 Laser induced fluorescence technique for environmental applications [9286-26]

9286 OC Interferometry within a resonant cavity with standing wave detection [9286-213]

9286 OD Scalable lidar technique for fire detection [9286-27]

MO.2.A

9286 OG Characterization of arbitrary fiber taper profiles with optical microscopy and image processing algorithms [9286-52]

$9286 \mathrm{OH}$ Experimental setup for electromagnetically induced transparency observation in hollowcore fibers [9286-119]

MO.2.B

9286 OK CW 1.06- $\mu \mathrm{m}$ pumped ytterbium-holmium co-doped all-fiber laser for $2.05 \mu \mathrm{m}$ [9286-232]

9286 ON Design of high-brightness TEMoo-mode solar-pumped laser for renewable material processing [9286-86] 
9286 OP Near-field resonant energy transfer between spherical quantum dots [9286-48]

$92860 \mathrm{O}$ On the electro-optical behavior of an OLED based on europium and the relationship with electrical transport phenomena [9286-160]

9286 OR Wavemeter uncertainty evaluation for the calibration of external cavity diode lasers [9286-37]

9286 OY Split-ring resonators manufactured on conductive layer by selective laser ablation [9286-136]

$9286 \mathrm{OZ}$ Effect of $\mathrm{CO}_{2}$ laser micromachining on physicochemical properties of poly(L-lactide) [9286-141]

928610 Monitoring the laser cleaning of granites by means of multispectral image analysis [9286-181]

928611 Excimer laser-induced incubation of poly(L-lactide) [9286-106]

928612 Fabrication of Fresnel microlens with excimer laser contour ablation [9286-124]

928615 Dichroism of stretched poly vinyl alcohol (PVA) foils containing pyridazinium ylids II [9286-176]

TU.1.A

928618 Limits of spectral resolution in optical measurements (Invited Paper) [9286-248]

9286 1A A numerical method for calculated spectral transmittance of wavelength filter in large band slits [9286-142]

TU.1.B

9286 1B Using single photons to improve fiber optic communication systems [9286-174]

9286 1C A brief review on quantum bit commitment [9286-150]

9286 IE Photon-pair generation in lossy waveguides [9286-32] 
TU.2.A

9286 IG A technique for the reconstruction of a map of continuous curves from interference fringes [9286-43]

$9286 \mathrm{1H}$ Use of laser speckle and entropy computation to segment images of diffuse objects with longitudinal motion [9286-46]

TU.2.B

9286 1K Investigation of PPSLT waveguides for applications in optical communication systems [9286-169]

9286 IL Advanced optical modulation and format conversion [9286-31]

9286 1M All-optical modulation format conversion from OOK to DP-QPSK based on fiber nonlinearity [9286-154]

TU.2.C

9286 IN Solid to liquid light: soliton dynamics using GPU computing (Invited Paper) [9286-140]

9286 IP High-power high-brightness solar laser approach for renewable $\mathrm{Mg}$ recovery from $\mathrm{MgO}$ [9286-87]

TU.3.A

9286 is Construction and validation of a Shack-Hartmann type aberrometer [9286-195]

9286 IT Personalized pseudophakic model [9286-24]

TU.3.B

9286 1X Optimal launch power prediction of a 100G PM-DQPSK dispersion-managed link with the Gaussian noise model [9286-153]

TU.P

9286 1Y Free space optical communications for ultrahigh-capacity PON system [9286-180]

928623 Conversion gain improvement of HBT-optoelectronic mixers using structural design alteration of device [9286-68]

928625 Real-time upper-body human pose estimation from depth data using Kalman filter for simulator [9286-227] 
928627 Parametric impairments analysis of all-optical format conversion techniques with a MZISOA [9286-51]

928628 Optics activity for hospitalized children [9286-60]

928629 Contact lenses fitting teaching: learning improvement with monitor visualization of webcam video recordings [9286-134]

9286 2D Hyphenated GC-FTIR and GC-MS techniques applied in the analysis of bioactive compounds [9286-209]

$92862 \mathrm{E}$ FEM simulation and experimental determination of the temperature profile of nanoparticles excited by laser radiation [9286-202]

$92862 \mathrm{~F} \quad$ Surface modification induced by UV nanosecond Nd:YVO 4 laser structuring on biometals [9286-183]

$92862 \mathrm{H} \quad$ Laser induced heating of PMMA microspheres for remote drug release: a FEM simulation model [9286-91]

$928621 \quad$ Reflection of light: a teaching and learning activity with primary school children [9286-204]

$92862 \mathrm{~J} \quad$ Why do shadows change length? An IBSE approach with primary school children [9286-208]

TU.4.A

9286 2K Visual imagery without visual perception: lessons from blind subjects [9286-240]

TU.4.B

$92862 \mathrm{Q}$ Plastic optical fibre sensor for Madeira wine monitoring [9286-30]

WE.1

$92862 T$ Intermolecular interactions in ternary solutions of some 1,2,4-triazolium ylids studied by spectral means [9286-172]

SEONS I

$92862 \mathrm{U}$ Sensors based on recycled optical fibers destroyed by the catastrophic fuse effect (Invited Paper) [9286-21] 
WE.2.A

9286 2X Light pollution: why should we care? (Invited Paper) [9286-101]

$92862 Y \quad$ Study of repeatability of an optical laser system for characterization of the paper fiber distribution and mass density [9286-59]

$92862 Z$ A new method to determine the optical rotatory dispersion of transparent crystalline layers [9286-175]

928630 Top lateral refraction and reflection of polarized light in lenses [9286-57]

WE.2.B

928635 Raman studies of tungsten yttrium tellurite glasses as high and broad gain media [9286-182]

928637 A versatile fluorescence lifetime imaging system for scanning large areas with high time and spatial resolution [9286-214]

SEONS III

$92863 \mathrm{~B}$ Analysis and design of a $1 \times 2$ ring resonator-based plasmonic switch [9286-7]

9286 3C A note on ultra-short pulses compression in silicon optical waveguides under fourth-order dispersion [9286-105]

WE.3

9286 3D Theoretical background of pulsed laser melting in liquid for submicron spherical particle fabrication (Invited Paper) [9286-243]

$92863 \mathrm{E} \quad$ Laser-induced breakdown spectroscopy (LIBS) technique for the determination of the chemical composition of complex inorganic materials [9286-81]

SEONS IV

9286 3J Mode conversion based on the acousto-optic effect for mode division multiplexed transmission [9286-113]

9286 3K Characteristics study of multimode directional coupler by elliptical point contacts and CMT [9286-98] 
$92863 \mathrm{M}$ Study of the stabilization of a semiconductor mode-lock laser using hybrid mode-lock and optical feedback [9286-130]

WEDNESDAY POSTER SESSION

$92863 \mathrm{P}$ Evaluation of polymerization shrinkage of resin cements through in vitro and in situ experiments [9286-84]

$92863 R \quad$ Guidance features at terahertz spectrum of microstructured fibers filled with silver rods [9286-102]

9286 3T Synthesis of gold nanoparticles for application as biosensors in engineering [9286-179]

$92863 \mathrm{U}$ Gas sensing using wavelength modulation spectroscopy [9286-192]

9286 3W Repeatability analysis on LPFGs written by a CO 2 laser [9286-99]

$92863 X$ Determination of Terfenol-D magnetostriction characteristics for sensor application using fiber Bragg grating [9286-90]

$92863 Y \quad F l u i d$ evaporation monitoring with suspended-core fibers [9286-201]

$92863 Z$ Wavefront shaping using a deformable mirror for focusing inside optical tissue phantoms [9286-96]

928640 Theoretical modeling of an U-shaped SPR fiber sensor in 1550-nm spectral range for sensing applications [9286-146]

$928641 \quad$ Ultrasensitive nanosensor based on silver nanoparticles to detect hydrogen peroxide [9286-205]

928642 Thermal monitoring of the thermoplastic injection molding process with FBGs [9286-220]

928643 Regeneration of FBGs during the HFCVD diamond-fiber coating process [9286-83]

928646 Remote curvature fiber sensors using core mismatch structures and OTDR based interrogation [9286-66]

928649 Electro-optical parameters in excited states of some spectrally active molecules [9286-178]

9286 4A Analysis of phase interrogated SPR fiber optic sensors with different bimetallic combinations [9286-222] 
TH.1.A

9286 4B Fiber optic cryogenic sensors for superconducting magnets and superconducting power transmission lines at CERN (Invited Paper) [9286-104]

9286 4C First setup of the optical fiber measuring system to monitoring structure health of nuclear power plant [9286-45]

9286 4D Preparation and measurement of TFBG based vibration sensor [9286-85]

TH.1.B

$92864 \mathrm{E} \quad$ Revisiting grating orientation effects on visual contrast sensitivity using optical interferometry [9286-148]

9286 4G Development of a micro PET system with improved spatial resolution through depth-ofinteraction measurement [9286-212]

$92864 \mathrm{H} \quad$ Study of gastric cancer samples using terahertz techniques [9286-93]

92864 Study of the fluorescence signal for gastrointestinal dysplasia detection [9286-54]

TH.1.C

9286 4K Performance analysis of multi-pump Raman+EDFA hybrid amplifiers for WDM systems [9286-103]

$92864 \mathrm{~L} \quad$ Reducing the complexity of digital nonlinear compensation for high-speed coherent optical communication systems [9286-203]

$92864 \mathrm{M}$ Clock and carrier recovery in high-speed coherent optical communication systems [9286-170]

TH.2.A

928640 Optical fiber sensors in arterial pulse waveform acquisition [9286-82]

$92864 \mathrm{Q}$ Fiber optic dosimeter with silicon photomultipliers [9286-211]

9286 4R Real-time dosimeter targeted to nuclear applications [9286-200]

TH.2.B

$92864 S$

Digitally controlling the 'twist' of light (Invited Paper) [9286-70] 
9286 4W Reinforced adaboost face detector using support vector machine [9286-230]

$92864 \mathrm{X}$ Resolution analysis in computational imaging with patterned illumination and single-pixel detection [9286-117]

$92864 Z$ Design and optimization of a spectrometer for spectral domain optical coherence tomography [9286-167]

928650 Experimental research of methods for clustering and selecting image fragments using spatial invariant equivalent models [9286-238]

928654 SLM-based optical simulator for dynamic speckle analysis [9286-123]

928658 Optical non-invasive 3D characterization of pottery of pre-colonial Paranaiba valley tribes [9286-219]

$92865 \mathrm{~B} \quad$ Fill-factor and performance optimization in bulk-heterojunction organic solar cells [9286-162]

9286 5C Influence of a bleaching post-exposure treatment in the performance of H-PDLC devices with high electric conductivity [9286-97]

\section{FR.1.A}

928651 On the impact of fiber-delay-lines (FDL) in an all-optical network (AON) bottleneck without wavelength conversion (Invited Paper) [9286-34]

9286 5J Energy-aware RWA for IP transport over WDM networks [9286-218]

$92865 \mathrm{~K}$ Efficiency analysis on platform over the top (OTT) to deploy content and applications (edutainment) in digital television on optical network link [9286-143]

FR.1.B

$92865 \mathrm{M}$ Optical response of fractal aggregates of polarizable particles [9286-28] 


\section{Authors}

Numbers in the index correspond to the last two digits of the six-digit citation identifier (CID) article numbering system used in Proceedings of SPIE. The first four digits reflect the volume number. Base 36 numbering is employed for the last two digits and indicates the order of articles within the volume. Numbers start with 00, 01, 02, 03, 04, 05, 06, 07, 08, 09, OA, OB...0Z, followed by 10-1Z, 20-2Z, etc.

Ab-Rahman, Mohd Syuhaimi, 3K Abramski, Krzysztof M., OY, OZ, 11, 12, 3E Abreu, Cátia, 21

Abreu, M. C., 4Q

Abreu, Manuel, 3M, 3W

Alberto, Nélia J., 2Q, 2U, 40, 42, 43

Albuquerque, André A., $1 \mathrm{~K}$

Almaguer, Citlalli, 28

Almeida, Álvaro J., 07, 1B, $1 \mathrm{C}$

Almeida, Joana, ON, 1P

Almeida, Luiz C. P., 3T

Almeida, Telmo P., 1X

Alves, Márcia Angelina, 58

Amado, Sofia B., 4L, 4M

Amaral, Maria E., $2 Y$

André, Paulo S., 07, 1C, 1X, 2U, $4 \mathrm{O}$

Antończak, Arkadiusz J., OY, OZ, 11, 12, 3E

Antunes, Paulo, 2Q, 2U, 40

Argibay-Losada, Pablo Jesus, $5 \mathrm{I}$

Arines, Justo, 29

Arsad, Norhana, 3K

Auguste, J.-L., $3 Y$

Avó, R., 06

Baierl, H., 3Y

Bajas, $H_{\text {., }} 4 \mathrm{~B}$

Bajko, M., 4B

Balderas-Mata, S. E., 15

Balle, S., 06

Baptista, J. M., 3U

Bará, Salvador, $2 X$

Barmenkov, Yuri O., OK

Bastos, José M., 40

Beixiga, Vasco, OD

Beléndez, Augusto, 5C

Belgacem, Mohamed N., $2 Y$

Belsley, Michael, 37

Benchea, Andreea Celia, 2T, 49

Berberova, Nataliya, 54

Bernardo, César, 37

Bértolo, Helder, 2K

Bilro, Lúcia, 2Q, 40

Blanco, J., OR

Borges, Joel, 5M

Brandão, Simão, 05

Breaban, IUliana, $2 Z$

Breglio, G., 4B

Buchta, Zdenek, 4C

C., Yunkoo, 4W

Cabral, Alexandre, 3M
Cambronero, Ferran, 28

Capela, Diana, $1 \mathrm{H}$

Cardoso, João, $1 \mathrm{H}$

Cardoso, Markos P., 3R

Cardozo da Silva, Jean Carlos, $3 X$

Carmelo Rosa, Carla, 2E, 4R, 4Z

Carneiro, Fatima, $4 \mathrm{H}$

Cartaxana, Paulo, 09

Carvalho Silva, Catia D., 4H

Carvalho, M. Inês, $\mathrm{OH}$

Castanheira, E. M. S., 4 I

Castanheira-Dinis, A., $1 T$

Castro Alves, D., 3M, 3W

Castro, I. F. C., 4G, 4Q

Cavaleiro, Albano, 5M

Chi, S., 25

Chiuchiolo, A., 4B

Číp, Ondřej, OC, 4C

Clemente, P., $4 \mathrm{X}$

Closca, Valentina, 2T, 49

Coelho, João M. P., 2E, 2H, 3W, $3 Z$

Coelho, L., 4A

Consales, M., 4B

Correia, Alexandre, 4R

Correia, Carlos, $1 \mathrm{H}$

Correia, P. M. M., 4G

Corte-Real, J. P., 4E

Costa, Ana P., 2 Y

Costa, Manuel F. M., 2I, 2J, 58

Costa, Pedro S., 4M

Cosutchi, Irina, $2 Z$

Cusano, A., 4B

C. W. A. Costa, João, 3R, 46

Delgado, Tamara, 28

de Matos Gomes, Etelvina, 37

de Morais Sousa, Kleiton, $3 X$

de O. Mendes, António, $2 Y$

de Sousa, Marco J., 46

Dias, J. M., $1 T$

Dimitriu, Dan Gheorghe, $2 Z$

Dionísio, Rogério P., 1L, 27

Diz-Bugarin, J., OR

Domingues, M. Fátima, $2 \mathrm{U}$

Dorohoi, Dana Ortansa, 15, 2T, 2Z, 49

Dorrío, B. V., OR

Drummond, Miguel V., 1K, 1M, 1X

Duarte, Daniel P., 40

Duarte, Vanessa C., IM

Dudley, A., 4S 
Dumitrascu, Irina, 15

Dumitrascu, Leonas, 15

Facão, Margarida, $\mathrm{OH}$

Falcão, António N., 4R

Farias, Heric D., OG

Felizardo, Rui, 09

Fernandes, Cindy S., 46

Fernandes, Gil M., OH, 3J

Fernández, Roberto, 5C

Ferreira, J., $3 \mathrm{U}$

Ferreira, Mario, $\mathrm{OH}$

Ferreira, R. A. S., $2 U$

Ferreira, Requicha, $1 \mathrm{H}$

Ferreira, Ricardo, 05, 1Y, 4M

Fewo Ibraid, S., 3C

Fiadeiro, Paulo T., 2Y, 4E

Figueiredo, J. M. L., 06

Fiorucci, M. Paula, 10, 2F

Flores, D., 3U

Flores-Arias, Maria T., 28

Forbes, A., 4S

Franco, A. P. G. O., 3P

Frazão, Orlando, 3U, 3Y, 46

Frias, Ana Rita, $2 \mathrm{U}$

Gabriel, Ana, $3 Z$

Gallego, Sergi, 5C

Gameiro, Carla, 09

Garcia-Sanchez, Angel, 28

Gargallo, Ana, 28, 29

Gentil Ferreira, A. F., Jr., 1 A

Ghodsi, Hamed, 23

Giordano, M., 4B

Gomes, J., 5B

Gomes, O. M. M., 3P

Gomes, Ricardo, $3 Z$

Gómez-Varela, Ana I., 28

Gonçalves, Hugo, 37

González-Nuñez, Hector, 28

Gosav, Steluta, 2D

Gouveia, Carlos J., 46

Graça, M.P. F., 35

Granja, Pedro L., 4H

Guerreiro, A., 1N, 4A

Guimarães, Marco C. C., 3T, 41

Guiomar, Fernando P., 4L, 4M

Helan, Radek, 4C, 4D

Henriques, M., 5J

Hirohashi, Junji, $1 \mathrm{~K}$

Holá, Miroslava, 0C

Hosseiny, Hamid, $4 Z$

Hrabina, Jan, OC

Humeau-Heurtier, Anne, $1 \mathrm{H}$

Irles, E., 4X

Isakov, Dmitry, 37

Ivanov, Branimir, 54

J., Jaeyoon, 4W

Jamier, R., $3 Y$

Janusas, Giedrius, $1 G$

Jardim Martini, Márcia M., 4K

Javaloyes, J., 06
Jelinek, Michal, 4C

Jorge, Pedro A. S., 46

K., Jaehong, 4W

Kaatuzian, Hassan, 08, 23, 3B

Kalinowski, Hypolito José, 3P, 43, 4K

Karam, L. Z., 3P

Kasalynas, Irmantas, 4H

Keshavarz Moazzam, Mostafa, 3B

Khashei, Hamidreza, 08

Kim, J., 25

Kir'yanov, Alexander V., OK

Kofané, T. C., 3C

Kozioł, Paweł E., OY, 0Z, 11, 12, 3E

Krasilenko, Vladimir G., 50

Lancis, J., $4 \mathrm{X}$

Lazar, Josef, OC

Łazarek, Łukasz K., OY, 0Z, 11, 12, 3E

Lazarev, Alexander A., 50

Lee, D., 25

Leitão, Cátia, 40

Leite, I. T., 4A

Leone Filho, R., 1A

Liang, Dawei, ON, $1 \mathrm{P}$

Liebold, Falk, 37

Lima, Mário, 1 Y

Lopes, Ana, 05

López Olazagasti, E., is

López, Ana J., 10, 2F

Lorentz, Katharina, 4R

Loura, Ricardo, $1 \mathrm{C}$

Lousã, Pedro, OD

Magalhães, Wagner, 58

Mandeng Mandeng, L., 3C

Mãocheia, Paulo, 05

Marques, Manuel B., 18

Márquez, Andrés, 5C

Martelli, Cicero, $3 \mathrm{X}$

Mateus, Paulo, 1C

Matos, Ana R., 09

Melniciuc-Puica, Nicoleta, 2T

Mendes, Tiago, 05

Mikel, Bretislav, 4C, 4D

Milaneze, Bárbara A., ЗТ

Minas, G., 4 I

Minkovich, Vladimir P., OK

Miranda Díaz, Lázaro J., 30

Moayyed, H., 4A

Molis, Gediminas, 4H

Monteiro, Fernando, 3W

Monteiro, Jorge M., $\mathrm{OH}$

Moreno, Gustavo A., 5K

Morosanu, Cezarina, 49

Mota, Pedro, OD

Moura, J. P., 3Y

Moutinho, L. M., 4Q

Muga, Nelson J., 07, 1B, 1C, 3J, 4M

Navarro-Fuster, Víctor, $5 \mathrm{C}$

Nespereira, Marta, 3W

Neto, Anselmo Frizera, 3T

Neto, Victor F., 42, 43 
Nikitovich, Diana V., 50

Nogueira, Breno V., 3T, 41

Nogueira, Rogério N., 1K, 1L, 1M, 1X, 27, 2Q, 40, 42,43

Noversa, Silvana, 2J

Novo, C., 2Q

Oliveira Silva, Catarina, $3 Z$

Oliveira, Jairo P., 3T, 41

Ortuño, Manuel, 5C

Oulehla, Jindřich, OC

Outumuro, I., OR

Paduraru, Nicoleta, 2D

Palevicius, Arvydas, $1 \mathrm{G}$

Palevicius, Paulius, $1 \mathrm{G}$

Palmieri, L., 4B

Park, C. H., 25

Park, C., 25

Pascual, Inmaculada, 5C

Paterno, Aleksander S., OG

Paunković, Nikola, 1C

Pavlović, Natasa B., IX

Peralta, L., 4Q

Pereira, Eduardo, 37

Pereira, L., OQ, 5B

Pereira, Paulo A. S., 5M

Pereira, Rui M. S., 5M

Pereira, Tânia, $1 \mathrm{H}$

Peres, Filipa C. R., OP

Perez, J. C., 4B

Piedade, Fernando, OD

Pimenta, S., 4l

Pinho, P., 5J

Pinto, Armando N., 07, OH, 1B, 1C, 1E, 3J, 4L, 4M

Pinto, João L., 2Q, 40

Pires, Vladimiro, 37

Piro, $0 ., 06$

Pontes, Maria José, 3T, 41, 4K

Pozo, S., 10

Prado, Adilson R., 3T, 41

Praisler, Mirela, 2D

Prezas, P. R., 35

Puche, William S., 5K

Pulido, C. A., 3P

Puttnam, Benjamin J., $1 \mathrm{~K}$

Pyatenko, Alexander, 3D

Ragulskis, Minvydas, $1 G$

Ramil, Alberto, 10, 2F

Ramírez Zavaleta, G., is

Ramza, Harry, 3K

Rastegar Pashaki, Elahe, 23

Rebordão, José M., 2H, 3M, 3W

Reis, Catarina, $3 Z$

Ribeiro, F., $1 \mathrm{~T}$

Ribeiro, J., 3U

Ribeiro, Moisés R. N., 3T, 41, 4K

Rivas, T., 10

Rocco Giraldi, Maria Thereza M., 46

Rodrigues, Cláudio, 05

Rodrigues, Francisco, 05

Rodrigues, Nuno F. G., 2E
Rodrigues, Silvia, $\mathrm{OH}$

Rodriguez, A. D., 4X

Romeira, B., 06

Roy, P., 3Y

Rusu, Cristina Marcela, 49

Sahin, Gokhan, 5I

Samantilleke, Anura, 37

Santos, G., OQ

Santos, J. L., 3U, 3Y, 4A

Santos, L. F., 4E

Santos, M. G., 5B

Santos, Pedro M. P., 4R

Schellenberg, Peter, 37

Sebem, Renan, OG

Seliuta, Dalius, $4 \mathrm{H}$

Serra, P. M., 4E

Shahpari, Ali, 1 Y

Shinada, Satoshi, $1 \mathrm{~K}$

Sierra, Javier E., 5K

Silva, Anderson O., 3R

Silva, Nuno A., 07, 1B, 1C, 1E, 1N

Simões, W., OQ

Smirnov, Georgi V., 5M

Sousa, Artur N., $1 Y$

Sousa, Marco J., 46

Stępak, Bogusz D., OY, 0Z, 11, 12, 3E

Stoica, Iuliana, 15

Stoykova, Elena, 54

Supian, L. S., 3K

Suresh Kumar, J., 35

Szustakiewicz, Konrad, 0Z, 11

Szymczyk, Patrycja, OY

Tajahuerce, E., 4X

Tavares, Ana, 05

Tchawoua, C., 3C

Teixeira, António L., 05, 1L, 1Y, 27, 4M, 5J

Tepichín Rodríguez, E., $1 S$

Tiburcio, Bruno D., OH

Trindade, A. J., 5B

Urban, Frantisek, Jr., 4D

Urban, Frantisek, Sr., 4D

Urbanowicz, Andrzej, 4H

Utkin, Andrei B., 09, OD

Vagner da Silva, Erlon, 3X

Valdivieso-González, L. G., is

Valencia, J. L., OR

Valusis, Gintaras, 4H

Varela, Paulo, 2l, 2J

Vasilevskiy, Mikhail I., OP, 37, 5M

Vaz, Filipe, 5M

Vaz, Pedro, 1H

Veloso, J. F. C. A., 4G, 4Q

Viegas, D., 4A

Vieira, Pedro, $3 Z$

Vilhena, Henrique, $2 \mathrm{H}$

Viveiros, D., $3 \mathrm{U}$

Volkers, R. E., 41

Wada, Naoya, $1 \mathrm{~K}$

Wahaia, Faustino, 4H

Walczakowski, Michał, oy 
Wójcik, Michał R., OY, 0Z, 11, 12, 3E

Yoom, Hosub, 25, 4W

Zandonay, Ricardo, $3 \mathrm{X}$

Zelinschi, Andreea Carmen, 2T, 49

Zelinschi, Beatrice Carmen, 15

Ziaie, Somayeh, $4 \mathrm{M}$

Proc. of SPIE Vol. 9286 928601-14

Downloaded From: https://www.spiedigitallibrary.org/conference-proceedings-of-spie on 26 Apr 2023 Terms of Use: https://www.spiedigitallibrary.org/terms-of-use 


\section{Conference Committees}

Conference General Chair

Manuel Filipe P. C. Martins Costa, Universidade do Minho (Portugal)

Conference Chair

Rogério Nunes Nogueira, Instituto de Telecomunicações

(Portugal)

Technical Chairs

António Baptista, Universidade do Minho (Portugal)

Gonçalo Figueira, Instituto Superior Técnico (Portugal)

José Figueiredo, Universidade do Algarve (Portugal)

Lúcia Bilro, Instituto de Telecomunicações (Portugal)

Orlando Frazão, INESC, Porto (Portugal)

Scientific Committee

Amparo Pons Martí, Universitat de València (Spain)

Ana Consortini, Università degli Studi di Firenze (Italy)

Anand Krishna Asundi, Optics and Photonics Society of Singapore

(Singapore)

Andrea Cusano, Università degli Studi del Sannio (Italy)

Andrew Moore, Herriot-Watt University (United Kingdom)

Andrés Márquez Ruiz, Universidad de Alicante (Spain)

Angel Augier Calderin, INSTEC (Cuba)

Angel I. Negueruela, Universidad de Zaragoza (Spain)

Angela M. Guzman, CREOL, The College of Optics \& Photonics, University of Central Florida (United States)

Asticio Vargas, CEFOP-Centro de Óptica y Fotónica (Chile)

Clementina Timus, INFLR (Romania)

Carlos Ferreira, SEDOPTICA—Sociedad Española de Óptica (Spain)

Clementina Timus, INFLR (Romania)

Cristiano M. B. Cordeiro, Universidade Estadual de Campinas (Brazil)

Daniel Malacara Hernández, Centro de Investigaciones en Óptica (Mexico)

Efraín Solarte Rodriguez, RCO—Red Columbiana de Óptica (Colombia)

Eric Rosas, Centro de Investigaciones en Óptica (Mexico)

Eugene Arthurs, SPIE

Gonçalo Figueira, Instituto Superior Técnico (Portugal)

Guillermo Baldwin, Pontificia Universidad Católica del Perú (Peru) 
Hai-Ning Cui, Nanjing University (China)

Hector Rabal, ClOP-Centro de Investigaciones Ópticas (Argentina)

Humberto Michinel, Universidade de Vigo (Spain)

Hypolito Kalinowski, Universidade Tecnológica Federal do Paraná

(Brazil)

James Wyant, The University of Arizona (United States)

Joaquín Campos Acosta, Instituto de Óptica, CSIC (Spain) and

SEDOPTICA-Sociedad Española de Óptica (Spain)

João Lemos Pinto, Universidade de Aveiro (Portugal)

João Manuel Tavares, Universidade do Porto (Portugal)

John Canning, The University of Sydney (Australia)

Jose Benito Vazquez-Dorrio, Universidade de Vigo (Spain)

José Figueiredo, Universidade do Algarve (Portugal)

José Luis Paz, CTOV (Venezuela)

José Ramiro Fernandes, Universidade de Trás-os-Montes e Alto Douro

(Portugal)

José R. Salcedo, (Portugal)

José Silva Gomes, Universidade do Porto (Portugal)

José Manuel Rebordão, Universidade Nova de Lisboa (Portugal)

Juan G. Darias Gonzalez, CEADEN-Centro de Aplicaciones

Tecnológicas y Desarrollo Nuclear (Cuba)

Luciano Alberto Angel-Toro, RCO-Red Columbiana de Óptica

(Colombia)

Luis Miguel Bernardo, Universidade do Porto (Portugal)

Luis Roso, Centro de Láseres Pulsados Ultracortos Ultraintensos (Spain)

Katrina Svanberg, University of Lund (Sweden)

Kiyofumi Matsuda, AIST (Japan)

Kim Chew Ng, Monash University (Australia)

Manuel Lopez-Amo, Universidad Pública de Navarra (Spain)

Manuel Melgosa Latorre, Universidad de Granada (Spain)

Marcelo Trivi, Universidad Nacional de Mar del Plata (Argentina)

Maria Josefa Yzuel, Universitat Autónoma de Barcelona (Spain)

Maria Luisa Calvo, Instituto de Crédito Oficial (Spain)

Maria Sagrario Millan, Universitat Politècnica de Catalunya (Spain)

Mário Vaz, INEGI, Porto (Portugal)

Maité Flores-Arias, Universidad de Santiago de Compostela (Spain)

Marta Ramos, Universidade do Minho (Portugal)

Maxim Tomilin, University of St. Petersburg (Russia)

Miguel Gonzalez Herraez, Universidad de Alcalá (Spain)

Mikiya Muramatsu, Universidade de São Paulo (Brazil)

Mikhail Vasilevski, Universidade do Minho (Portugal)

Mourad Zghal, STO (Tunisia)

Mustafa Erol, Bozok University (Turkey)

Pablo Artal, Universidad de Murcia (Spain)

Paulo Tavares, Universidade do Porto (Portugal)

Pedro Andrés, RIAO, Universitat de València (Spain)

Radu Chisleag, Technical University of Bucharest (Romania) 
Ramón Rodríguez-Vera, Centro de Investigaciones en Óptica (Mexico)

Raul Rangel, Academia Mexicana de Óptica, CICESE (Mexico)

Rastogi Pramod, École polytechnique Fédérale de Lausanne

(Switzerland)

Roger Ferlet, Université de Paris (France)

Salvador Bará, Universidad de Santiago de Compostela (Spain)

Tomas Catunda, Universidade de São Paulo (Brazil)

Sabry Abdel-Mottaleb, Ain-Shams University (Egypt)

Sun Tong, City University (United Kingdom)

Toyohiko Yatagai, SPIE Vice President (Japan)

Waclaw Urbanczyk, Wroclaw University of Technology (Poland)

Zuqing Zhu, University of Science and Technology of China (China)

Program Committee

Alexandre Cabral (Portugal)

Alicia Fernandéz Oliveras (Spain)

Anand Krishna Asundi (Singapore)

Angel Augier Calderin (Cuba)

Angela M. Guzman (USA)

Ana Maria Rocha (Portugal)

António Batista (Portugal)

Clementina Timus (Romania)

Efraín Solarte Rodriguez (Colombia)

Eric Rosas (Mexico)

Gonçalo Figueira (Portugal)

Hai-Ning Cui (China)

Humberto Michinel (Spain)

Ireneu Dias (Portugal)

João M. P. Coelho (Portugal)

João Manuel Tavares (Portugal)

Joaquim Carneiro (Portugal)

Jose Benito Vazquez-Dorrio (Spain)

José Figueiredo (Portugal)

José Luis Paz (Venezuela)

José Luís Santos (Portugal)

José Manuel Baptista (Portugal)

José R. Salcedo (Portugal)

José Silva Gomes (Portugal)

Lúcia Bilro (Portugal)

Luis Miguel Bernardo (Portugal)

Kim Chew Ng (Australia)

Manuel Filipe P. C. Martins Costa (Portugal)

Manuel Joaquim Marques (Portugal)

Mário Lima (Portugal)

Naoya Wada (Japan) 
Nélia Alberto (Portugal)

Orlando Frazão (Portugal)

Rogério Nunes Nogueira (Portugal)

Organizing Committee Vice-Chairs

Ana Maria Rocha, Instituto de Telecomunicações (Portugal)

Nélia Alberto, Instituto de Telecomunicações (Portugal)

Organizing Committee Members

Álvaro Almeida, Instituto de Telecomunicações (Portugal)

André Albuquerque, Instituto de Telecomunicações (Portugal)

Gil Fernandes, Instituto de Telecomunicações (Portugal)

Telmo Almeida, Instituto de Telecomunicações (Portugal)

Vanessa Cunha Duarte, Instituto de Telecomunicações (Portugal)

Session Chairs

1 Plenary Session I

Manuel Filipe P. C. Martins Costa, Universidade do Minho (Portugal)

Rogério Nunes Nogueira, Instituto de Telecomunicações (Portugal)

2 Mo.1.a

Moisés Ribeiro, Universidade Federal do Espírito Santo (Brazil)

3 Mo.1.b

Alexander Pyatenko, National Institute of Advanced Industrial Science and Technology (Japan)

$4 \quad$ Mo.2.a

Lúcia Bilro, Instituto de Telecomunicações (Portugal)

5 Mo.2.b

Helder Crespo, Universidade do Porto (Portugal)

6 Plenary Session II

Armando Pinto, Instituto de Telecomunicações (Portugal)

$7 \quad$ Tu.1.a

Daniel Albach, Helmholtz-Zentrum Dresden-Rossendorf e.V. (Germany)

$8 \quad$ TU.1.b

Rogério Dionísio, Instituto Politécnico de Castelo Branco (Portugal) 
9 Tu.2.a

Manuel Ryser, Universität Bern (Switzerland)

10 Tu.2.b

Prince Anandarajah, Dublin City University (Ireland)

11 Tu.2.C

Angela Dudley, CSIR National Laser Centre (South Africa)

12 Plenary Session III

António Baptista, Universidade do Minho (Portugal)

13 Tu.3.a

Helder Bertolo, Instituto Superior de Engenharia de Coimbra (Portugal)

14 Tu.3.b

Peter Andrekson, Chalmers University of Technology (Sweden)

15 Tu.4.a

Paulo Fiadeiro, Universidade da Beira Interior (Portugal)

$16 \quad$ Tu.4.b

Aleksander S. Paterno, Universidade Federal de Santa Catarina (Brazil)

17 Plenary Session IV

Yoshinari Awaji, National Institute of Information and Communications Technology (Japan)

18 We.1

Manuel P. Graça, Universidade de Aveiro (Portugal)

19 SEONS I

Orlando Frazão, INESC Porto (Portugal)

20 We.2.a

Manuel Marques, Universidade do Porto (Portugal)

21 SEONS II

José Luís Santos, Universidade do Porto (Portugal)

22 We.2.b

Mikhail Vasilevskiy, Universidade do Minho (Portugal)

23 SEONS III

Mário Lima, Instituto de Telecomunicações (Portugal) 
24 We.3

Paulo André, Universidade de Lisboa (Portugal)

25 SEONS IV

Maria Medeirios, Instituto de Telecomunicações (Portugal)

26 We.4

Salvador Bará, Universidad de Santiago de Compostela (Spain)

27 OSA Student Chapter Session

Rogério Nunes Nogueira, Instituto de Telecomunicações, Aveiro (Portugal)

28 Th.1.a

Jean Silva, Universidade Tecnológica Federal do Paraná (Brazil)

29 Th.1.b

Pedro Pombo, Universidade do Aveiro (Portugal)

$30 \quad$ Th.1.c

Benjamin Puttnam, National Institute of Information and Communications Technology (Japan)

31 Plenary Session $\mathrm{V}$

Manuel Filipe P. C. M. Costa, Universidade do Minho (Portugal)

32 Th.2.a

Gonçalo Figueira, Universidade Técnica de Lisboa (Portugal)

33 Th.2.b

Humberto Michinel, Universidad de Vigo (Spain)

34 Plenary Session VI

John Barrera-Ramírez, Universidad de Antioquia (Colombia)

$35 \quad$ Fr.1.a

Lutz Rapp, Coriant (Germany)

36 Fr.1.b

Ana Maria Rocha, Instituto de Telecomunicações (Portugal)

37 Closing Session

João Lemos Pinto, Universidade de Aveiro (Portugal) 


\section{Introduction}

The Second International Conference on Applications of Optics and Photonics ran very successfully in an exciting, friendly, and rewarding way. Nearly two hundred participants openly shared ideas and experiences discussing the remarkable and exciting recent developments in optics and photonics, and about preparing new development paths and cooperation projects.

The conference was organized 26-30 May 2014, at the Meliã Ria Hotel \& Spa in Aveiro, Portugal. It was another major international event organized by the Portuguese Society for Research and Development of Optics and Photonics, SPOF-Sociedade Portuguesa para a Investigação e Desenvolvimento em Óptica e Fotónica (www.optica.pt, contact@spidof.pt). The Portuguese Optics and Photonics Society was established in November 2009-reflecting the development of the scientific and technological research in optics and photonics in Portugal over previous decades, and our main goal of promoting the development of optics and photonics in general and particularly in Portugal. Coming from all Portugal's continental and Atlantic autonomous regions, SPOF's membership is increasing in almost all fields of pure and applied research both at the university and research institutions, but also in the industry services and education.

The conference was organized to foster the establishment of the widest range of cooperation projects and relationships with colleagues and institutions from all around the world, while increasing the external visibility of Portugal, optics and photonics research. This year, the conference also hosted the Symposium on Enabling Optical Networks and Sensors (SEONS), which allowed for dedicated sessions in the field of optical networks and sensors.

The success of the conference was only possible with the commitment of the vast majority of Portuguese optics and photonics scientists and the endorsement support and active participation of the leadership of the most important international scientific optics societies and several national societies, committees, and boards.

The 612 authors were from 39 different countries-reflecting the international character of this conference. We are pleased to stress that roughly $50 \%$ of the participants were students and young researchers.

The scientific program included seven plenary sessions, three short courses, 24 invited presentations, 83 oral presentations, 86 posters, and a special session dedicated to entrepreneurship in optics and photonics. 
We would like to thank the endorsers/sponsors and all participants who have made this conference a success!

Manuel Filipe P. C. Martins Costa Rogério Nunes Nogueira 